\title{
Configural learning in humans: The transverse patterning problem
}

\author{
ROBERT S. ASTUR and ROBERT J. SUTHERLAND \\ University of New Mexico, Albuquerque, New Mexico
}

\begin{abstract}
Most learning theorists concur that some sort of configural representation system is required for animals to solve certain compound discriminations. Transverse patterning (A+ vs. B-, B+ vs. $\mathrm{C}-, \mathrm{C}+$ vs. $A^{-}$) is one example of a problem that requires a configural solution. It has been reported in the past that adult humans are unable to solve this problem (Berch \& Israel, 1971; Franks, 1976). We investigated the generality of these reports. Moreover, we examined whether a stepwise approach to training this problem would facilitate learning and at what stage in training subjects adopt a configural strategy. We found that college-aged adults had little difficulty solving the transverse patterning problem, and that their learning was greatly facilitated by using the stepwise approach described by Alvarado and Rudy (1989). Moreover, we found that subjects seem to adopt a configural strategy when faced with ambiguous stimulus pairs even when an elemental strategy would suffice. These results provide insight into how humans solve configural problems and also suggest some direct tests of the role of the hippocampus in configural associations.
\end{abstract}

Traditionally, learning theorists have explained in various ways how animals solve problems that consist of compound elements. Elemental theorists posit that in order to solve such a problem, the strengths of each element in the compound are manipulated, and these elements are then algebraically summed to determine the responding strategy (Hull, 1943; Rescorla \& Wagner, 1972). Configural theorists contend that such problem-solving behaviors are controlled by a compound representation that is a single separate stimulus distinct from its components, and that this compound's strength determines responding (Kehoe \& Gormezano, 1980; Rescorla, 1972; Rudy \& Wagner, 1975). Alternatively, Pearce (1987) would explain these behaviors by allocating the control of the response solely to the compound representation, whereas Holland (1984) would argue that response control by the elements and the compound depends on the type of the elements as well as the stimulus' level of representation.

Nonetheless, despite such discrepancies among theorists' views of the exact role and representation of these configural cues, it seems apparent that elemental theories are insufficient to explain certain problems that have no linear solution and hence cannot be solved by simple elemental strategies. For example, neither negative patterning $(\mathrm{A}+, \mathrm{B}+, \mathrm{AB}-)$ nor feature-neutral discrimina-

This research was supported in part by the Department of Psychology, QUAD-L foundation, and Minority Biomedical Research Support Program of the University of New Mexico. The authors are indebted to Jerry Rudy and two anonymous reviewers for their invaluable comments on an initial draft of this paper and to Maria Ortiz, Andrea Baca, Jennifer Dungan, and Laura Rickert for their expert technical assistance in these experiments. Correspondence should be addressed to R. S. Astur, Department of Psychology, Logan Hall, University of New Mexico, Albuquerque, NM 87131 (e-mail: rsax@unm.edu). tions $(\mathrm{AC}+\mathrm{C}-, \mathrm{AB}-\mathrm{B}+)$ can be explained by a simple summing of the strengths of the individual elements of the compounds. Hence, it seems that some sort of configural representation system must be available for an animal to solve these problems.

In 1952, Spence presented a learning problem, called transverse patterning, which cannot be solved on the basis of responding to or avoiding a single cue. Specifically, the problem is as follows: $A+$ versus $B-, B+$ versus $\mathrm{C}-, \mathrm{C}+$ versus $\mathrm{A}-$. Note that each stimulus is ambiguous, and that the only way to solve this problem is to attend to the relationships between the stimuli. Again, in this case, no linear solution will allow an animal to solve this problem. Rather, the problem requires that the animal utilize a configural solution.

It has been reported that adult humans often are unable to solve this problem (Berch \& Israel, 1971; Franks, 1976). This is unexpected for a number of reasons. Berch and Israel reported that if they provided an additional dimension such as color that will allow for the formation of a linear solution (e.g., the red triangle is always correct, the blue square is always incorrect, etc.), all of their subjects were able to solve the transverse patterning problem. However, if their stimuli were all the same color, only half of their subjects could solve the problem. This suggests that their subjects were not using a configural strategy to solve the problem. Moreover, rats (Alvarado \& Rudy, 1989, 1992), monkeys (Alvarado, Wright, \& Bachevalier, 1995), chimpanzees (Thompson, 1953), pigeons (Couvillon \& Bitterman, 1996; Wynne, 1996), and children over 4.5 years (Rudy, Keith, \& Georgen, 1993) have all been able to solve the problem.

There are some critical procedural differences between the latter and the former experiments. The experiments with rats, pigeons, chimpanzees, or children in- 
volve a stepwise training of the problem. Specifically, subjects were first trained on $\mathrm{A}+$ versus $\mathrm{B}-$. After training on that problem, $\mathrm{B}+$ versus $\mathrm{C}$ - was introduced concurrently with $\mathrm{A}+$ versus $\mathrm{B}-$. Finally, after training in this phase, $\mathrm{C}+$ versus $\mathrm{A}-$ was introduced concurrently with the other two problems. Only in this final phase are all the stimuli ambiguous. This seems to be an important procedural difference, because Rudy et al. (1993) claimed that rats were unable to solve the problem if a stepwise procedure was not utilized. A second difference is that Berch and Israel (1971) used a maximum of 90 trials to reach criterion. However, no maximum was used in the experiments with nonhumans. It may well be that the subjects that did not reach criterion were approaching it when the trial maximum was reached.

These differences notwithstanding, Alvarado and Rudy (1992) conducted a number of experiments that suggest that rats used a relational strategy in Phase $2(\mathrm{~A}+$ vs. B-, $\mathrm{B}+$ vs. $\mathrm{C}-$ ) when a simple associative strategy would suffice. Note that in this phase of testing, an animal could always respond to $\mathrm{A}$ and always avoid $\mathrm{C}$ and still perform perfectly, and hence avoid using a relational strategy. However, their data suggest that this was not the case. Physiologically, this is especially interesting, because if indeed the hippocampus is a critical structure involved in relational/configural learning as Sutherland and Rudy claim (1989; see also Rudy \& Sutherland, 1995), then animals with hippocampal damage should utilize different strategies to solve this second phase.

Adult humans certainly can solve a number of configural problems such as negative patterning $(A+, B+$, $A B-$ ), oddity problems ( $A B+, B A+B)$, and biconditional discriminations ( $\mathrm{Cl}$ : $\mathrm{A}+$ vs. $\mathrm{B}-; \mathrm{C} 2$ : $\mathrm{A}-$ vs. $\mathrm{B}+$ ). However, the way in which these problems are solved has not yet been characterized. In this paper, we address a number of issues surrounding the transverse patterning problem in an attempt to obtain more information about the manner in which this configural problem is solved.

\section{EXPERIMENT 1}

Initially, it was important to determine whether humans can solve the transverse patterning problem when immediately tested on the full problem. For the sake of comparison, we contrasted their performance on this problem with that of three linear discriminations whereby the relationships between the stimuli are nonessential to solve the discriminations.

\section{Method}

Subjects. Sixty-four undergraduates ( 23 males, 41 females; average age, 23.1 years) at the University of New Mexico participated in this experiment for course credit. All subjects had normal or corrected-to-normal vision. Forty-three subjects were placed in the transverse patterning condition, and the other 21 were placed in the linear discrimination condition.

Apparatus. Stimuli were presented on a computer monitor. Each stimulus consisted of variously colored random polygons overlaid upon each other. This was an attempt to minimize subjects' naming the stimuli. Choices were made by using a joystick to move a cursor on the screen to contact a stimulus.

Procedure. After signing informed consent forms, the subjects were told that they would see a pair of stimuli on the screen, and that they would be allowed to choose only one stimulus on each trial. The subjects heard a pleasant rising tone if they chose the correct stimulus and a low buzzing tone if they chose the incorrect stimulus. They were given exposure to these feedback tones before testing. An intertrial interval of $2 \mathrm{sec}$ was used. Side of presentation and order of presentation were pseudorandomly arranged. For the transverse patterning problem, the stimulus pairs were as follows:

$$
\begin{array}{ll}
\text { A } & \text { B- } \\
\text { B }+ & \text { C- } \\
\text { C }+ & \text { A- }
\end{array}
$$

For the three linear problems, the stimulus pairs were as follows:

$$
\begin{array}{cc}
\text { U+ } & \text { V- } \\
\text { W+ } & \text { X- } \\
\text { Y } & \text { Z- }
\end{array}
$$

The subjects were required to score $13 / 15$ correct on each problem concurrently before reaching criterion. If after 420 trials criterion was not reached, the session was terminated.

\section{Results}

On the transverse patterning problem, of the 43 subjects, 39 reached criterion. For the 4 who did not reach criterion, the average number of errors was 211.2. On the linear discriminations, all 21 subjects reached criterion. Subjects made significantly fewer errors $[t(58)=3.68$, $p<.001]$ and significantly fewer trials to criterion [ $t(58)$ $=3.34, p<.001]$ when solving the linear discriminations than when solving the transverse patterning problem (see Figure 1).

\section{Discussion}

As can be seen from these data, nearly all subjects were able to solve the transverse patterning problem within 110 trials. However, there were 4 subjects who were unable to solve it. Upon debriefing, it became apparent that they had tried to use an across-trials strategy such as " $A$ was on the left last trial, so $C$ on the right next trial will be correct." Obviously, such a strategy is ineffective. It may be that increasing the intertrial interval would eliminate such a strategy. Another method might be initially to provide an irrelevant discrimination for a number of trials so that subjects might solve this and no longer assume that the stimulus side or previous spatial arrangement were relevant factors. All subjects had very little difficulty solving the linear discriminations, suggesting that the configural task was more difficult than the linear, or elemental, task.

\section{EXPERIMENT 2}

It was critical to see whether the stepwise testing of the transverse patterning problem would facilitate learning of the problem as Rudy et al. (1993) claimed. Again, for the sake of comparison, we included three linear discriminations. 


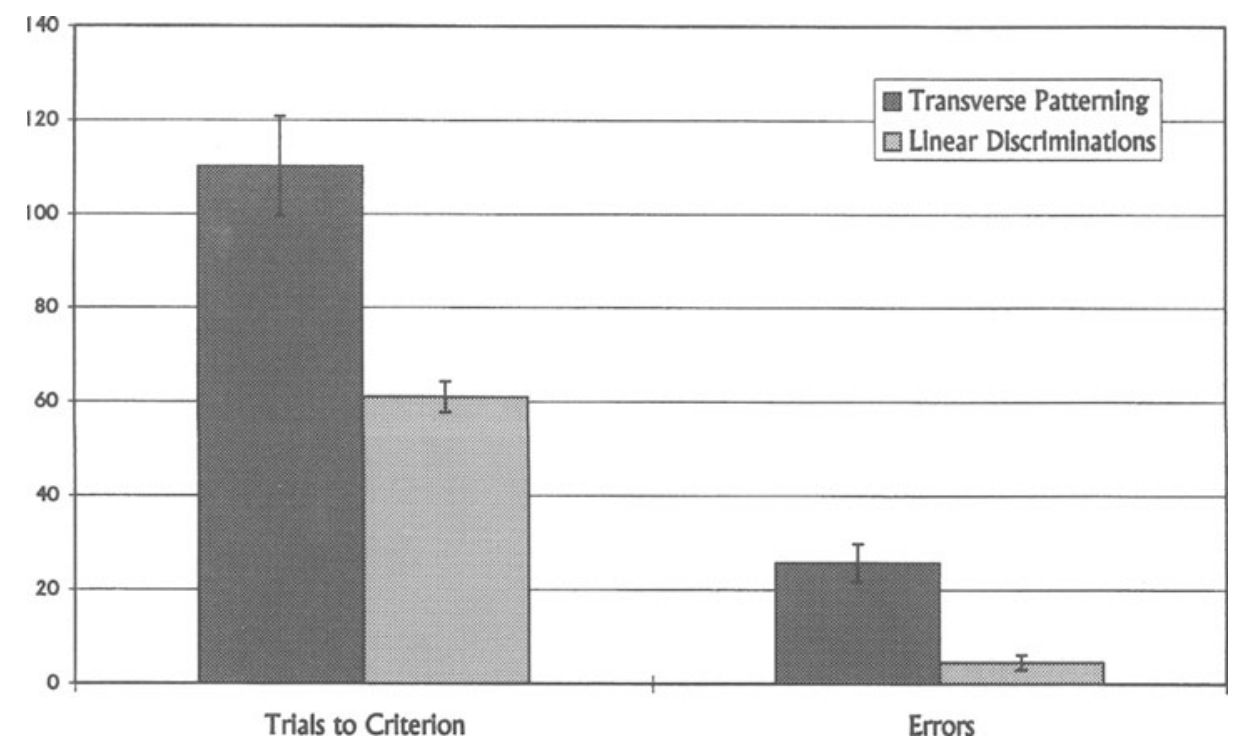

Figure 1. Trials to criterion and number of errors for the transverse patterning problem and for the linear discriminations. Subjects performed significantly fewer trials to criterion and made significantly fewer errors when solving the linear discriminations.

\section{Method}

Subjects. Forty-four undergraduates (14 males, 30 females; average age, 22.8 years) at the University of New Mexico participated in this experiment for course credit. All subjects had normal or corrected-to-normal vision. All subjects completed both types of problems. None of these subjects had participated in Experiment 1.

Apparatus. The apparatus was the same as that in Experiment 1.

Procedure. The procedure was the same as that in Experiment 1. Each subject was tested on each problem set, and the order of presentation was counterbalanced. The testing was performed in phases (see Table 1). The subjects were required to score $13 / 15$ correct concurrently on each problem before advancing to the next phase.

\section{Results}

All 44 subjects reached criterion. Comparison of the total number of errors across phases in this experiment with the total number of errors in Experiment 1 shows that subjects made significantly fewer errors with the stepwise training for both transverse patterning $[t(81)=$ $-5.41, p<.001]$ and the linear discriminations $[t(61)=$ $-2.42, p<.01]$. With the stepwise method, subjects still made significantly fewer errors when solving the linear discriminations than when solving the transverse patterning problem $[t(41)=4.69, p<.001]$ (see Figure 2 ).

Table 1

Testing Phases in Experiment 2

\begin{tabular}{|c|c|c|c|c|}
\hline \multirow{2}{*}{$\begin{array}{c}\text { Phase } \\
1\end{array}$} & \multicolumn{2}{|c|}{$\begin{array}{l}\text { Transverse } \\
\text { Patterning }\end{array}$} & \multicolumn{2}{|c|}{$\begin{array}{c}\text { Linear } \\
\text { Discriminations }\end{array}$} \\
\hline & $\mathrm{A}+$ & B- & $\mathrm{U}+$ & $\mathrm{V}-$ \\
\hline \multirow[t]{2}{*}{2} & $A+$ & B- & $U+$ & $V-$ \\
\hline & $\mathrm{B}+$ & C- & $\mathrm{W}+$ & $X-$ \\
\hline \multirow[t]{3}{*}{3} & $A+$ & B- & $\mathrm{U}+$ & $\mathrm{V}-$ \\
\hline & $\mathrm{B}+$ & $\mathrm{C}-$ & $w+$ & $X-$ \\
\hline & $\mathrm{C}+$ & $\mathrm{A}-$ & $\mathrm{Y}+$ & $Z-$ \\
\hline
\end{tabular}

\section{Discussion}

As can be seen from these data, the stepwise approach to the transverse patterning problem greatly facilitates performance. The average number of errors was 26.2 in Experiment 1 as opposed to just 2.1 errors in Experiment 2 . These data are in agreement with Rudy et al. (1993), who claimed that this procedure is often easier and more appropriate for nonhuman species. The trialsto-criterion measure does not always seem to reflect the average number of errors in our experiments appropriately. This is because once a subject has reached criterion on a problem, it is desirable to keep presenting those problems so that the ambiguity of the yet-to-be-solved problems continues to be the same. Hence, subjects have more trials to criterion than would be expected if each problem was dropped once criterion was reached. Note, however, that this does not mean that subjects could perform poorly overall and still reach criterion. The subjects were required concurrently to reach $13 / 15$ on all three problems in order to reach criterion.

\section{EXPERIMENT 3}

Given the results of Experiments 1 and 2, it seems that at least under some circumstances, humans have little difficulty in solving the transverse patterning problem. However, given that these results are in disagreement with those of Berch and Israel (1971), it was of importance to attempt to replicate their findings with the same stimuli, the same criterion, and the same trial maximum as they used. This would help us ascertain whether some of the subjects' being unable to solve the transverse patterning problem was due to a difference in apparatus, ge- 


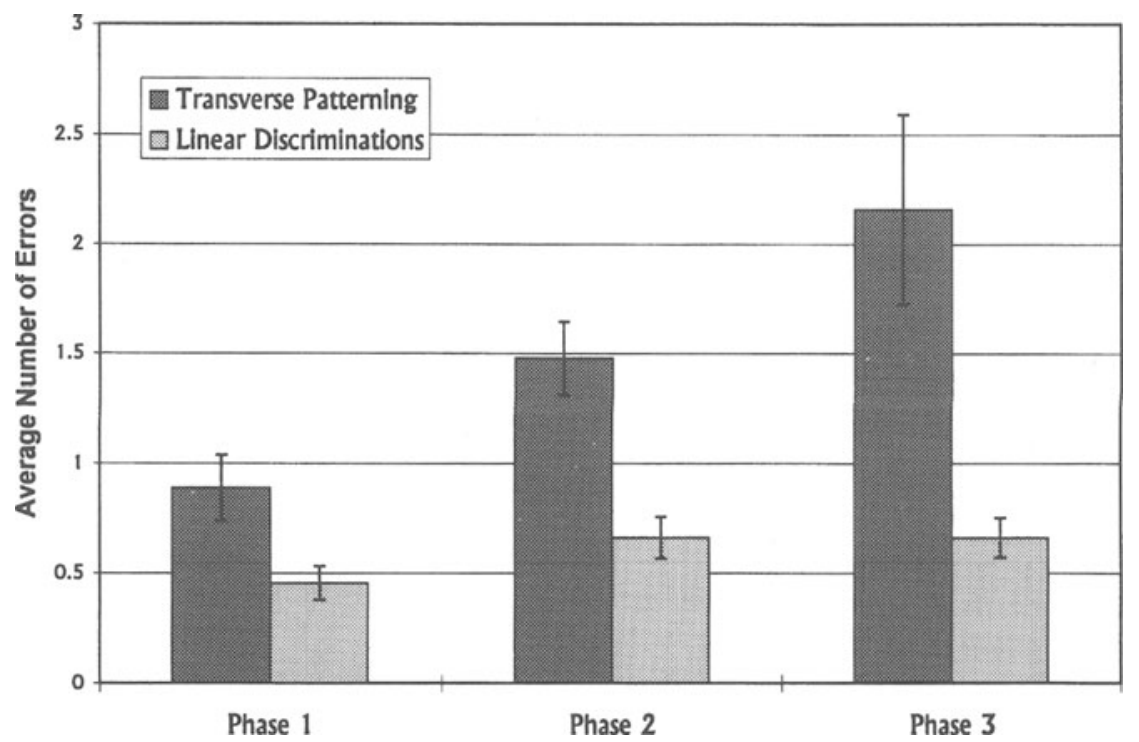

Figure 2. Errors for each test phase for stepwise transverse patterning and linear discriminations. Subjects still made significantly fewer overall errors when solving the linear discriminations than when solving the transverse patterning problem.

ography, decade of testing, or paradigmatic factors such as stimuli, criterion, or trial maximum.

\section{Method}

Subjects. Nineteen undergraduates ( 5 males, 14 females; average age, 21.6 years) at the University of New Mexico participated in this experiment for course credit. All had normal or corrected-tonormal vision. None of these subjects had participated in Experiment 1 or 2 .

Apparatus. Pairs of stimuli were presented on a computer monitor. The subjects responded by pressing a key on the left side of the keyboard to choose the leftmost stimulus and by pressing a key on the right side of the keyboard to choose the rightmost stimulus. Three stimuli were used: a circle, a square, and a triangle. All the stimuli were colored white.

Procedure. After they had signed informed consent forms, the subjects were told, "In this task, you will be presented with pairs of geometric shapes. You are to choose one stimulus in the pair. After your choice, you will be told whether that choice is "correct" or "wrong." Your goal is to get as many correct as possible." These instructions were paraphrased from Berch and Israel (1971). In addition, the appropriate keys to use on the keyboard were marked with colored tape so that the subjects knew which keys to use. Again, to replicate Berch and Israel, trials were presented in 15 blocks of 6 trials, with each trial type being presented once within these 6 trials in a pseudorandom order. The subjects were run until they reached a criterion of $12 / 12$ correct in succession or until they reached the 90 -trial maximum. The pairings were as follows:

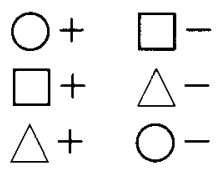

\section{Results}

Ten out of the 19 subjects did not reach criterion after 90 trials. Of the 9 who did, the average number of errors was $9.9 \pm 5.3$. The average trials to criterion were $39.1 \pm$ 18.0. Of the 10 who did not reach criterion, the average number of errors was $42.8 \pm 9.8$.

\section{Discussion}

These data are in agreement with those of Berch and Israel (1971). Specifically, after only 90 trials, using these stimuli and this criterion, it does not seem that the transverse patterning problem is readily solvable by the majority of adult undergraduates.

\section{EXPERIMENT 4}

It seems apparent that the majority of humans can readily solve the transverse patterning problem if given a generous trial maximum, particularly if the problem is presented in a stepwise manner. However, it is not clear when, under the stepwise procedure, subjects will begin to use a configural strategy. One way to solve the problem is that in Phase 1, subjects could always respond to $\mathrm{A}+$ and ignore $\mathrm{B}-$. Then, in Phase 2 , they could always avoid $\mathrm{C}-$ and still not attend to $\mathrm{B}+$. This would be an elemental approach to solving the first two phases of the transverse patterning problem. Another way would be to use a configural strategy immediately in Phase 2 and realize that $\mathrm{B}$ is incorrect when paired with $\mathrm{A}$ but correct when paired with $C$.

To address these alternatives, we used an experimental design similar to that used by Alvarado and Rudy (1992) to address the same question in rats. Specifically, the subjects were presented with a modified version of transverse patterning: Phase 1, A+ versus $\mathrm{B}-$; Phase 2 , $\mathrm{A}+$ versus $\mathrm{B}-, \mathrm{C}+$ versus $\mathrm{D}-$; Phase $3, \mathrm{~A}+$ versus $\mathrm{B}-, \mathrm{C}+$ versus $\mathrm{D}-$, and $\mathrm{D}+$ versus $\mathrm{A}-$. Note that none 
Table 2

Testing Phases in Experiment 4

\begin{tabular}{ccc}
\hline & \multicolumn{2}{c}{ Modified } \\
Phase & Transverse Patterning \\
\hline 1 & A & B- \\
2 & C + B- \\
& D - \\
3 & C & D- \\
& D + & A- \\
\hline
\end{tabular}

of the stimuli are ambiguous until Phase 3 , when the valances ( \pm ) associated with $A$ and $D$ are reversed. This problem can be compared to the standard transverse patterning problem used in Experiment 2: Phase 1, $\mathrm{A}+\mathrm{B}-$; Phase $2, \mathrm{~A}+\mathrm{B}-$ and $\mathrm{B}+$ versus $\mathrm{C}-$; Phase $3, \mathrm{~A}+\mathrm{B}-$, $\mathrm{B}+$ versus $\mathrm{C}-$, and $\mathrm{C}+$ versus $\mathrm{A}-$, where ambiguity was first introduced in Phase 2. If the subjects were using an elemental strategy to solve Phase 2 of the standard transverse patterning problem in Experiment 2, they should have made as many errors on the $\mathrm{C}+$ versus $\mathrm{A}$ - problem in Phase 3 as subjects who solved the modified transverse patterning problem would make on the $\mathrm{D}+$ versus $\mathrm{A}-$ problem in Experiment 4. However, if subjects trained on the standard transverse patterning problem in Experiment 2 were using a configural strategy to resolve the ambiguity introduced in Phase 2, this strategy should have transferred to Phase 3 and enabled them to have made fewer errors than would subjects who solved the modified problem in Experiment 4, who encountered ambiguity for the first time in Phase 3.

Thus in Experiment 4, subjects were presented with the modified transverse patterning problem. The results of their performance in Phase 3 were compared with the performance of the subjects trained on the standard transverse patterning problem in Experiment 2.

\section{Method}

Subjects. Twenty-two undergraduates ( 5 males, 17 females; average age, 22.7 years) at the University of New Mexico participated in this experiment for course credit. All subjects had normal or corrected-to-normal vision. None of the subjects had participated in any of the other experiments.

Apparatus. The apparatus was the same as that in Experiment 1.

Procedure. The procedure was the same as that of Experiment 1. However, the testing was performed in the phases shown in Table 2. The subjects were required to score $13 / 15$ correct on each problem concurrently before advancing to the next phase. Note in Table 2 that Phase 3 was a complete reversal of the valence of $A$ and $D$ as learned in Phase 2.

\section{Results}

All 22 subjects reached criterion. The critical comparison is whether subjects treated $\mathrm{C}+\mathrm{A}-$ in Phase 3 from Experiment 2 in the same way as subjects treated $\mathrm{D}+\mathrm{A}-$ in Phase 3 from Experiment 4. The subjects made significantly more errors in the $\mathrm{D}+\mathrm{A}-$ phase than in the $\mathrm{C}+\mathrm{A}-$ phase from Experiment $2[t(64)=2.76$, $p<.01$ ] (see Figure 3). These results are consistent with the hypothesis that, even though Phase 2 of the standard transverse patterning problem can be solved by using elemental associations, humans appear to switch to the configural association strategy at this stage. Had they still employed elemental associations in Phase 2, subjects who solved the standard transverse patterning problem should have made as many errors on the $C+$ versus $A-$ problem in Phase 3 as subjects trained on the modified problem made on the $D+$ versus $A$ - problem.

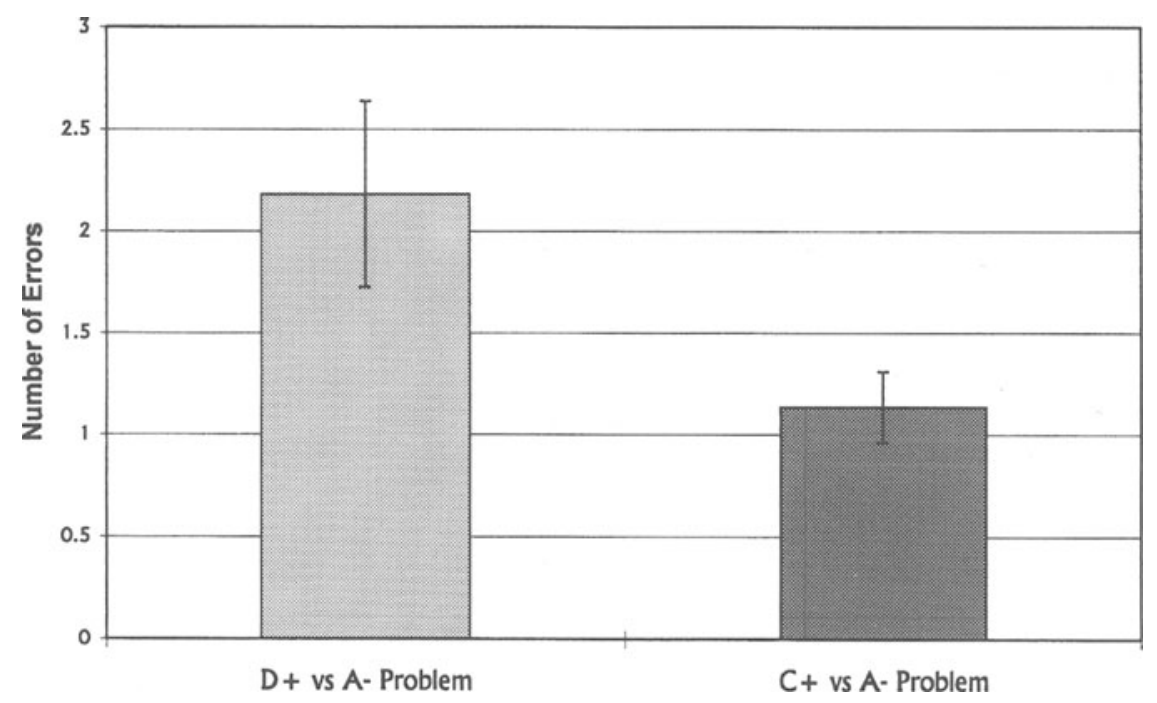

Figure 3. Number of errors for a complete valence reversal versus errors in Phase 3 of stepwise transverse patterning. Subjects made significantly fewer error in the transverse patterning condition than in the reversal condition, suggesting that they were using a configural strategy to solve Phase 2. 
As was pointed out by an anonymous reviewer, it may be inappropriate to make this comparison across experiments, because the subjects in Experiment 2 were tested at a different time in the semester than were those in Experiment 4 . In an attempt to reconcile these differences in time of testing, we have analyzed the Phase 1 performance data for all subjects in Experiment 2 and Experiment 4 . Note that for both experiments, Phase 1 is the $\mathrm{A}+\mathrm{B}-$ discrimination. We have found that these subjects did not differ in number of errors $[t(64)=0.352$, $p>.05]$, trials to criterion $[t(64)=0.15, p>.05]$, or response times $[t(64)=0.10, p>.05]$ in this phase. These data suggest that these groups seem to have been relatively homogeneous, at least as far as these measures allow us to detect.

\section{Discussion}

Given that the subjects showed more disruption by the complete reversal of the valences of $A+D-$ in Phase 3 of this experiment than in Phase 3 of Experiment 2, it seems that subjects were using a configural strategy in Phase 2 of the transverse patterning problem, even though it was not necessary to do so. These data coincide with Alvarado and Rudy's (1992) report that rats also used a configural strategy in Phase 2. Note, however, that whereas it seems that subjects were using a configural strategy in Phase 2, it is not clear what type of configural strategy they were using. One possibility could be that the subject thinks " $\mathrm{A}$ is correct when paired with $\mathrm{B}$, but $B$ is correct when paired with C." However, another option would be to think that "B can have two different values." Currently, it is not apparent which of these two strategies was employed. However, both are relational strategies: That is, both require the subject to pay attention to the relationships between the paired stimuli to respond correctly.

An anonymous reviewer pointed out that subjects still might adopt an "approach $\mathrm{A}+$, avoid $\mathrm{C}-$ " strategy, and then use this information to abstract a strategy such as "In this task, I have to do something other than just pay attention to $\mathrm{S}+$," which would then be beneficial in Phase 3. This indeed is true, but this still is not the strict avoid $\mathrm{C}-$, approach $\mathrm{A}+$ strategy. Rather, it involves an abstract strategy whereby subjects realize that their responses may differ, depending on the cues. Nonetheless, this is a valid alternative, and given the present experiments, we cannot distinguish which of these alternative strategies was being used. This is the topic of additional experiments from our laboratory.

The critical point from Experiment 4 is that subjects were not merely thinking in Phase 2, "Always respond to stimulus A and always avoid stimulus C," as their oneand-only strategy. Instead, our data indicate that a nonelemental strategy was being used even though it was not necessary. Additionally, one must keep in mind that despite the similarities in performance in Phase 1 between the subjects in Experiments 2 and 4, one should be cau- tious about interpreting these results, because the subjects in Experiment 2 were tested at an earlier point in the semester than were those in Experiment 4, and there might have been unforeseen factors inherent in this design that influenced these results.

\section{GENERAL DISCUSSION}

A number of important implications can be derived from this series of experiments. First, it seems apparent that most college-aged humans have little difficulty in solving the transverse patterning problem when placed immediately in the full problem. Whereas 4 out of 43 subjects did not solve the problem after 420 trials, it seems that their strategies could have been modified properly by introducing an irrelevant problem (e.g., $\mathrm{X}+$ $\mathrm{Z}-$ ) to change their inappropriate assumptions about the task (e.g., the assumption that side of presentation was a relevant factor) before introducing the full transverse patterning problem. Second, in support of Rudy et al. (1993), with the stepwise approach, subjects made significantly fewer errors in solving this problem and did so in fewer trials. Moreover, every subject was able to solve the problem. It is not clear what advantage is gained by using the stepwise approach. It may be that the stepwise approach serves to eliminate many incorrect assumptions that subjects might adopt, such as trying to solve the problems on the basis of stimulus location.

In support of Berch and Israel (1971), it seems that 90 trials are insufficient for one to solve the transverse patterning problem. However, this does not mean that subjects are unable to adopt the proper configural strategy to solve it. In fact, if we retroactively apply a 90 -trial limit to our subjects in Experiment 1, we will note that only 20/43 subjects would have solved the problem at that point. Nonetheless, by 110 trials on the average, $39 / 43$ subjects were able to adopt the appropriate configural strategy and solved the problem. In fairness to Berch and Israel, the main focus of their study was not whether or not subjects could solve the transverse patterning problem; rather, they were interested in what types of stimulus dimensions subjects would utilize to solve the problem. Those interests notwithstanding, their data and those of Franks (1976) have somehow led to a belief that adult humans have difficulty in solving the transverse patterning problem. This is certainly not the case.

Third, it is interesting that subjects adopted a nonelemental strategy in Phase 2 even though their previous elemental strategy would have sufficed. These data suggest not only that subjects are capable of using nonelemental strategies, but that when faced with stimulus ambiguity, subjects actually seem to prefer to switch to a nonelemental strategy even though it is not necessary. These data meld well with Rudy and Sutherland's (1995) configural association theory that increasing stimulus ambiguity increases the reliance on nonele- 
mental associations that a subject must use. Interestingly, whereas Rudy and Sutherland point out that in Phase 2, subjects could have both elemental and configural associations, subjects chose to switch to a nonelemental strategy to solve it. However, as of now, it is not apparent which strategy was being used in this phase.

These data can be used to test certain properties of how configural associations are related to hippocampal function. For example, as mentioned above, Rudy and Sutherland (1995) claim that increasing ambiguity between the stimuli should increase the reliance on configural associations to solve the problem at hand. Their claim is that the hippocampus is the major brain structure that allows these configural associations to disambiguate the stimulus compounds. Hence, not only would their theory predict that animals with hippocampal damage would be impaired in the full transverse patterning problem (as has been shown in rats by Alvarado \& Rudy, 1989 ), but moreover, their theory would predict that these animals would be unable to use a configural strategy in Phase 2 of the transverse patterning problem. To our knowledge, this has yet to be tested. Additionally, these data indicate that configural learning by rats shares some characteristics with those of humans and are in general theoretical agreement with Pearce and Wilson (1990), who state that "animals use configural information when solving a discrimination even though they do not need to do so" (p. 260).

In summary, college-aged humans have little problem in solving the transverse patterning problem when presented immediately with the full problem. However, solving this problem is greatly facilitated with the use of the stepwise approach as described by Rudy et al. (1993). Moreover, humans adopt a configural strategy when faced with ambiguous stimulus pairs even when an elemental strategy would suffice. These results are important in not only shedding light on how humans solve this particular configural problem, but also allowing for direct tests of the role of the hippocampus in configural associations.

\section{REFERENCES}

Alvarado, M. C., \& Rudy, J. W. (1989). The transverse patterning problem, configural processes and the hippocampus. Society for Neuroscience Abstracts, 15,610 .

Alvarado, M. C., \& RudY, J. W. (1992). Some properties of configural learning: An investigation of the transverse-patterning problem.
Journal of Experimental Psychology: Animal Behavior Processes, 18, 145-153.

Alvarado, M. C., Wright, A. A., \& Bachevalier, J. (1995). Monkeys with early hippocampal formation lesions are impaired on the transverse patterning problem. Society for Neuroscience Abstracts, 21, 1494.

BERCH, D. B., \& IsRAEL, M. (1971). Solution of the transverse patterning problem: Response to cue-cue relations. Psychonomic Science, 23, 383-384.

Couvillon, P. A., \& Bitterman, M. E. (1996). Transverse patterning in pigeons. Animal Learning \& Behavior, 24, 410-422.

FRANKS, G. J. (1976). The transverse patterning problem with ten-yearolds and college students. Unpublished doctoral dissertation, West Virginia University.

Holland, P. C. (1984). Origins of behavior in Pavlovian conditioning. In G. H. Bower (Ed.), The psychology of learning and motivation (Vol. 18, pp. 129-173). San Diego: Academic Press.

Hull, C. L. (1943). Principles of behavior. New York: AppletonCentury-Crofts.

KeHOE, E. J., \& GoRMEZANo, I. (1980). Configuration and combination laws in conditioning with compound stimuli. Psychological Bulletin, 87, 351-378.

Pearce, J. M. (1987). A model of stimulus generalization for Pavlovian conditioning. Psychological Review, 94, 61-73.

Pearce, J. M., \& Wilson, P. N. (1990). Configural associations in discrimination learning. Journal of Experimental Psychology: Animal Behavior Processes, 16, 250-261.

ResCoRlA, R. A. (1972). "Configural" conditioning in discrete-trial bar pressing. Journal of Comparative \& Physiological Psychology, 79, 307-317.

Rescorla, R. A., \& WAgner, A. R. (1972). A theory of Pavlovian conditioning: Variations in the effectiveness of reinforcement and nonreinforcement. In A. H. Black \& W. F. Prokasy (Eds.), Classical conditioning II: Current research and theory (pp. 64-99). New York: Appleton-Century-Crofts.

Rudy, J. W., KeITH, J. R., \& GeORGEN, K. (1993). The effect of age on children's learning of problems that require a configural association solution. Developmental Psychobiology, 26, 171-184.

Rudy, J. W., \& Sutherland, R. J. (1995). Configural association theory and the hippocampal formation: An appraisal and reconfiguration. Hippocampus, 5, 375-389.

RUDY, J. W., \& WAGNER, A. R. (1975). Stimulus selection in associative learning. In W. K. Estes (Ed.), Handbook of learning and cognitive processes (Vol. 2, pp. 269-304). Hillsdale, NJ: Erlbaum.

SPENCE, K. W. (1952). The nature of response in discrimination learning. Psychological Review, 59, 89-93.

SUTHERLAND, R. J., \& RUDY, J. W. (1989). Configural association theory: The role of the hippocampal formation in learning, memory, and amnesia. Psychobiology, 17, 129-144.

THOMPSON, R. (1953). Approach-avoidance in an ambivalent object discrimination problem. Journal of Experimental Psychology, 45, 341344.

WYNNE, C. D. L. (1996). Transverse patterning in pigeons. Behavioural Processes, 38, 119-130.

(Manuscript received August 4, 1997; revision accepted for publication November 24, 1997.) 